\title{
An Electron Capture Detector Based on a Non-Radioactive Electron Source
}

\author{
Erik Bunert, Ansgar T. Kirk, Jens Oermann, Stefan Zimmermann \\ Leibniz Universität Hannover, Inst. of Electrical Engineering and Measurement Technology, Dept. of Sensors and \\ Measurement Technology, Appelstr. 9A, 30167 Hannover, Germany \\ E-mail: bunert@geml.uni-hannover.de
}

\begin{abstract}
:
Gas chromatographs with electron capture detectors are widely used for the analysis of electron affine substances such as pesticides or chlorofluorocarbons. Being able to achieve limits of detection in the low pptv-range, electron capture detectors are the most sensitive detector available for such compounds. Based on their operating principle, they require free electrons at atmospheric pressure, which are usually generated using a $\beta$-decay. However, the use of radioactive materials leads to regulatory restrictions regarding the purchase, operation and disposal. Here, we present an electron capture detector based on a non-radioactive electron source, which is not subject to these limitations and offers further advantages such as adjustable electron density and energy.
\end{abstract}

Key words: Electron capture detector (ECD), gas chromatography, GC detector, non-radioactive electron source.

\section{Introduction}

Since their advent more than 55 years ago [1], electron capture detectors (ECD) have become the most important gas chromatography (GC) detector for the detection of electron affine compounds. Apart from the introduction of pulsed operation [2], their basic setup has remained unchanged. Although many attempts have been made to replace the radioactive source used to generate thermalized electrons by ionizing the carrier gas [3], only the pulsed helium discharge has seen some degree of practical use.

In other applications, for example ion mobility spectrometry, radioactive electron sources have been successfully replaced by non-radioactive electron sources. A compact, hermetically sealed electron source developed at our institute [4] is shown in Fig. 1. It generates free electrons through thermionic emission inside a vacuum cavity, which are then accelerated and transmit through a silicon nitride membrane of typically $300 \mathrm{~nm}$ thickness to atmospheric pressure. By varying the acceleration voltage and filament heating current, the electron energy and density can be adjusted. Furthermore, a control grid in front of the filament allows switching the emitted electron beam on and off. This enables a fast pulsed mode with adjustable electron pulse widths down to a few nanoseconds. The emission current can be controlled both in continuous electron emission mode and in pulsed mode $[4,5]$.

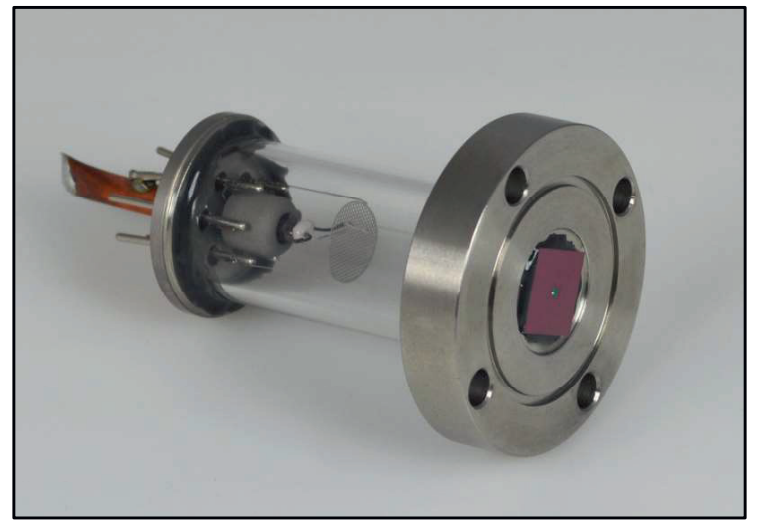

Fig. 1. Hermetically sealed non-radioactive electron source.

Using this non-radioactive electron source for an ECD avoids the disadvantages of its radioactive counterparts, while allowing comparable limits of detection (LoD). Furthermore, this nonradioactive electron capture detector can be operated in pulsed mode for an optimized sensor response, comparable to the pulsed discharge ECD $[6,7]$. Here we present first fundamental investigations regarding the analytical performance of an ECD equipped with our nonradioactive electron source.

\section{Experimental}

For first experimental investigations, our nonradioactive electron source is attached to a small ionization chamber manufactured from polyether ether ketone (PEEK). The electron source consists of a hot filament and a control grid and is sealed with a $300 \mathrm{~nm}$ thin silicon nitride 
membrane. The membrane is $1.5 \mathrm{~mm} \times 1.5 \mathrm{~mm}$ and transparent for electrons with sufficient electron energy [5]. On the opposite side of the ionization chamber, a Faraday plate detector is placed. Here, we designed two different configurations of the ionization chamber. In the first setup with orthogonal gas flow the carrier gas containing the analyte passes between the electron source and the Faraday detector as shown in Fig. 2 (a). In the second setup with axial gas flow the carrier gas containing the analyte flows from the Faraday detector towards the electron source as shown in Fig. 2 (b).

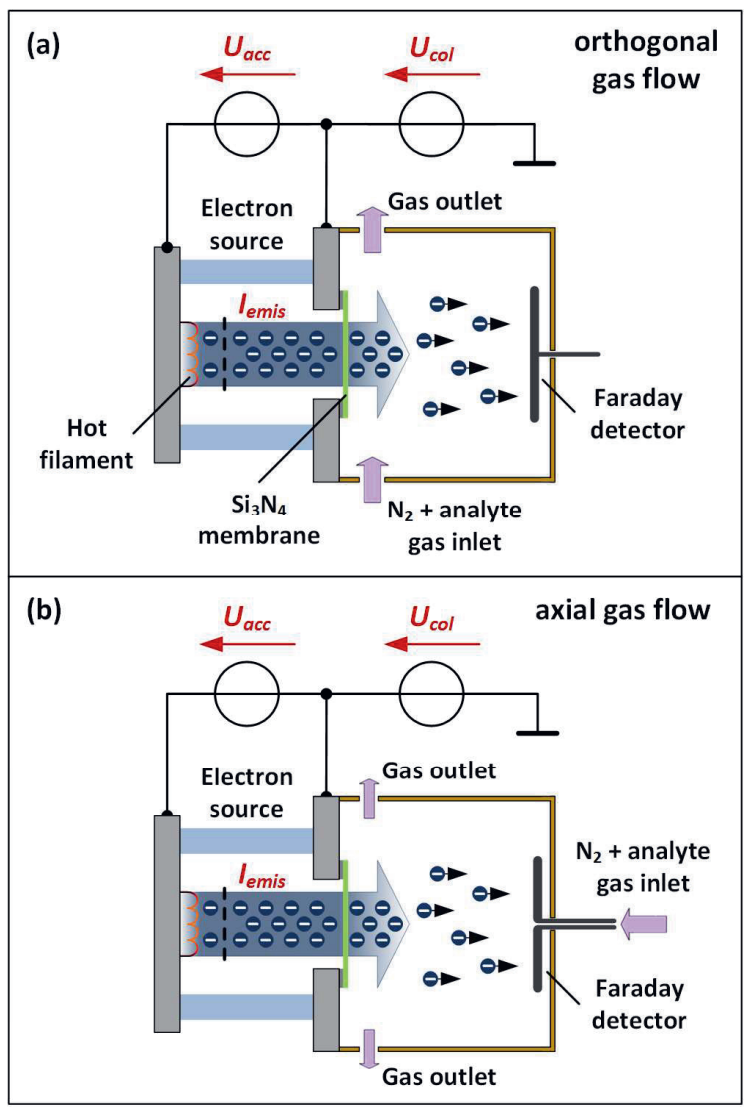

Fig. 2. Schematic of our electron capture detector with non-radioactive electron source with orthogonal (a) and axial (b) gas flow.

To adjust the kinetic electron energy, the acceleration voltage $U_{\text {acc }}$ between the hot filament and the acceleration electrode of the electron source can be varied between $8 \mathrm{kV}$ and $12 \mathrm{kV}$, which generates high kinetic electrons with energies between $4.5 \mathrm{keV}$ and $9.6 \mathrm{keV}$ after transmission through the membrane [5]. Adjusting the filament current in the range of $150 \mathrm{~mA}$ to $180 \mathrm{~mA}$ results in electron emission currents of $l_{\text {emis }}=10 \mathrm{nA}$ to $l_{\mathrm{emis}}=40 \mathrm{nA}$. These primary electrons with a penetration depth of about $0.5 \mathrm{~mm}$ at $U_{\text {acc }}=8 \mathrm{kV}$ to $2.2 \mathrm{~mm}$ at $U_{\text {acc }}=12 \mathrm{kV}$ in nitrogen ionize the nitrogen molecules in the ionization chamber by electron impact ionization and generate secondary electrons. By applying a constant collector voltage ( $\mathrm{DC}$ mode) of $U_{\text {col }}=3 \mathrm{~V}$ between the acceleration electrode of the electron source and the Faraday detector, the generated electrons can be collected at the Faraday detector, which constitutes a constant detector current. This detector current is amplified by our transimpedance amplifier [8] and measured by a Keysight 34461A multimeter. Higher collector voltages are not suitable, because the resulting absolute detector currents exceed the measuring range of $2 \mathrm{nA}$ of our existing amplifier. However, due to its low noise, this amplifier is still preferable to other designs.

The carrier gas containing the analyte flows with a constant flow of $30 \mathrm{mls} / \mathrm{min}$ through the ionization chamber, which is comparable to the values stated in the literature for gas chromatography (GC) with ECD, operating in DC mode [9]. Now, the electron-capturing compounds in the carrier gas can react with the free, thermalized electrons, leading to an decreased detector current [10].

For generating a constant analyte vapor concentration, the analyte is filled into a permeation vial heated to $35^{\circ} \mathrm{C}$ in a permeation oven. The oven is purged with a constant flow of $600 \mathrm{mls} / \mathrm{min}$ of nitrogen. An adjustable fraction of this gas is diluted with pure nitrogen for generating different analyte concentrations in the carrier gas. For preliminary characterization of both setups, we used 1,1,2-trichloroethane as analyte, purchased from Sigma Aldrich (CAS: 79-00-5).

\section{Results and discussion}

First, a constant 1,1,2-trichloroethane concentration of $50 \mathrm{ppb}_{\mathrm{v}}$ was used, and the decrease of the detector current, caused by the analyte reactions with the free electrons, which corresponds to the signal amplitude, was recorded to investigate the influence of the emission current, i.e. the generated electron density, on the maximum achievable sensitivity. The measurement results in Fig. 3 show that there are no significant differences in sensitivity between the axial and the orthogonal setup. However, increasing the electron emission current from $10 \mathrm{nA}$ to $40 \mathrm{nA}$ leads to an increased sensitivity by a factor of 3 . Unfortunately, higher emission currents cannot be investigated at the moment, as the resulting higher absolute detector currents are out of measuring range of our transimpedance amplifier. Thus, the best sensitivity for both setups is reached with an emission current of $40 \mathrm{nA}$. 


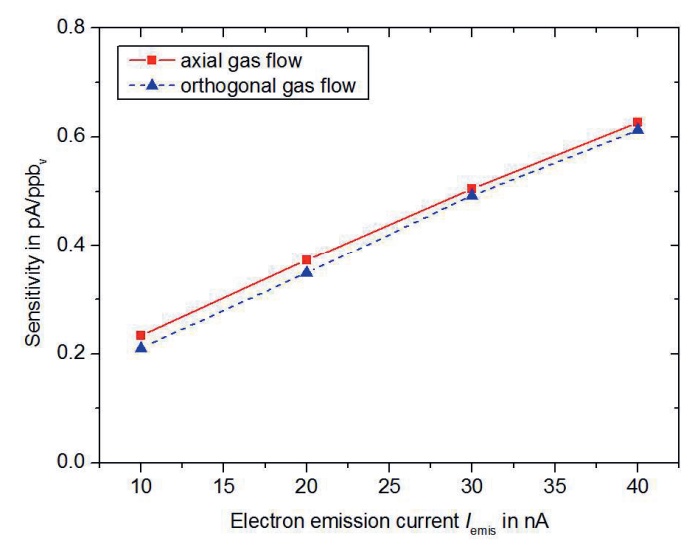

Fig. 3. Sensitivity over electron emission current from $10 n A$ to $40 n A$ with $50 p p b_{v}$ 1,1,2-trichloroethane concentration. The electron source acceleration voltage is $U_{\text {acc }}=10 \mathrm{kV}$.

Furthermore, the influence of the electron acceleration voltage, and thus the kinetic energy of the emitted electrons on the sensitivity were investigated. An increased acceleration voltage leads to an increased penetration depth of the emitted high kinetic electrons [5] and hence to a larger reaction region, which might improve the sensitivity. However, with lower kinetic energies, the electrons are thermalized in a shorter time. This is important for the reaction with electroncapturing analytes and can also lead to an improved sensitivity. Therefore, appropriate measurements are performed for both setups with a constant 1,1,2-trichloroethaneconcentration of $50 \mathrm{ppb}_{\mathrm{v}}$. The corresponding results, which are shown in Fig. 4, are measured with the highest possible emission current of $40 \mathrm{nA}$, while sweeping the acceleration voltage from $U_{\text {acc }}=8 \mathrm{kV}$ to $U_{\mathrm{acc}}=12 \mathrm{kV}$.

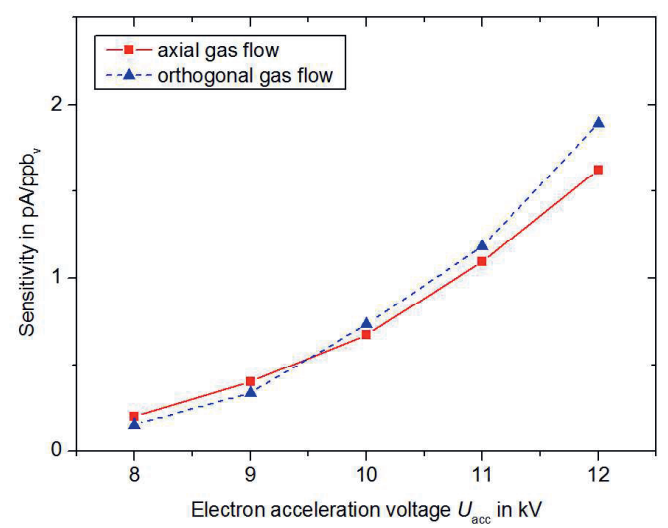

Fig. 4. Sensitivity over electron acceleration voltage from $8 \mathrm{kV}$ to $12 \mathrm{kV}$ with $50 \mathrm{ppb}_{\mathrm{v}}$ 1,1,2-trichloroethane concentration. The electron emission current is constantly controlled to $I_{\text {emis }}=40 n A$.

Here, the sensitivity increases with higher electron acceleration energies, which confirms the assumption that the higher penetration depth leads to a larger reaction region. Furthermore, electrons with higher kinetic energies can ionize more nitrogen molecules which generates more secondary electrons. These results suggest that the maximum sensitivity can be achieved by the highest acceleration voltage of $U_{\text {acc }}=12 \mathrm{kV}$.

However, not the sensitivity alone but rather a high signal-to-noise ratio is important for good LoD. Therefore, the SNR is most suitable for the comparison of the two described setups, depending on the electron emission current and the electron acceleration voltage. To determine the SNR for the described parameter sweeps, the noise of the detector signal is determined for a measuring time of $200 \mathrm{~ms}$ without analytes.

The influence of the emission current on the maximum achievable SNR for a 1,1,2trichloroethane concentration of $50 \mathrm{ppb}_{\mathrm{v}}$ and an acceleration voltage of $U_{\text {acc }}=10 \mathrm{kV}$ is shown in Fig. 5. The signal amplitude increases significantly with higher emission currents, while the noise just slightly increases. Thus, the best SNR is reached at an emission current of $l_{\text {emis }}=40 \mathrm{nA}$ for both setups.

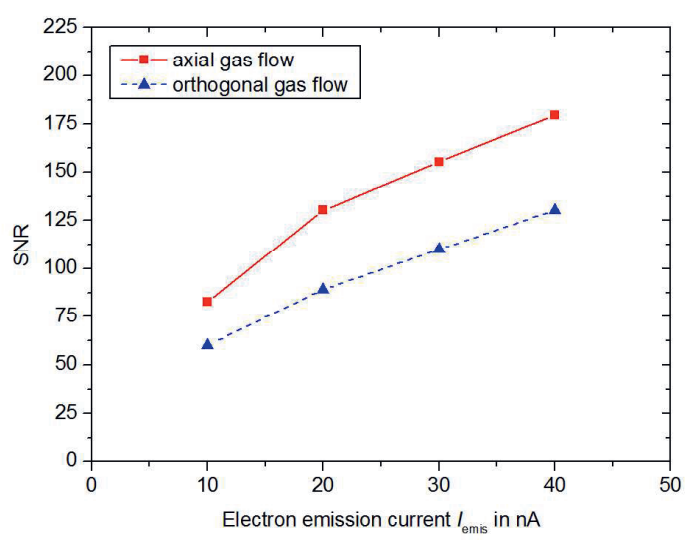

Fig. 5. SNR over electron emission current from $10 \mathrm{nA}$ to $40 \mathrm{nA}$ with an applied collector voltage of $3 \mathrm{~V}$ with $50 \mathrm{ppb}_{v}$ 1,1,2-trichloroethane concentration. The electron source acceleration voltage is $U_{a c c}=10 \mathrm{kV}$.

However, the SNR of the axial gas flow setup is significantly higher than the SNR of the orthogonal gas flow setup for each emission current. For example, at an emission current of $40 \mathrm{nA}$, the SNR of the axial setup is 175 and the SNR of the orthogonal setup is 130. A possible explanation for this can be that not all generated analyte ions can be flushed out by the gas flow, but drift towards the detector because of the applied collector voltage. This increases the statistical noise on the detector current, but the sensitivity is not significantly changed by the small number of ions lost this way. However, the axial gas flow transports the analyte ions in the opposite direction of the electrical field with a slightly higher force than the electrical force by the applied collector voltage. Thus, only the 
electrons but no analyte ions are detected by the Faraday detector.

Based on this, an increased acceleration voltage and thus an increased penetration depth of the emitted electrons and an increased number of generated secondary electrons should increase the detected noise by the statistical behavior of the ionization process. Therefore, the SNR for acceleration voltages between $U_{\mathrm{acc}}=8 \mathrm{kV}$ and $U_{\text {acc }}=12 \mathrm{kV}$ are determined for both setups. The resulting curves for the SNR, which are shown in Fig. 6, are measured with a constant emission current of $I_{\text {emis }}=40 \mathrm{nA}$.

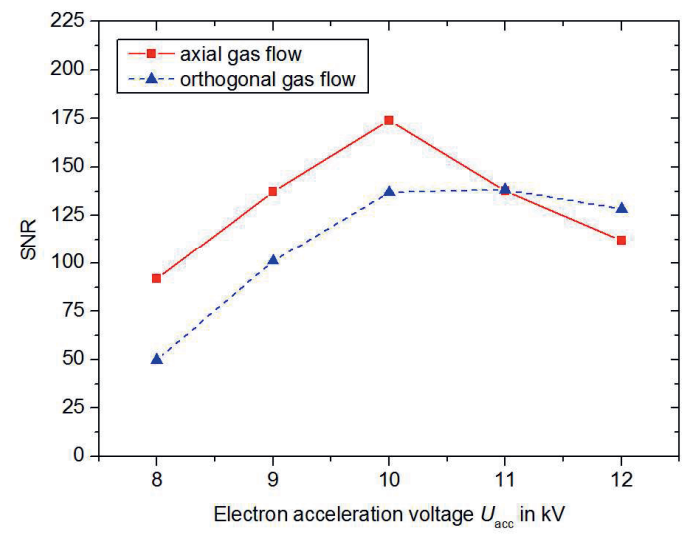

Fig. 6. SNR over acceleration voltage from $8 \mathrm{kV}$ to $12 \mathrm{kV}$ with an applied collector voltage of $3 \mathrm{~V}$ and $50 \mathrm{ppb}_{v}$ 1,1,2-trichloroethane concentration. The electron emission current is constantly controlled to $l_{\text {emis }}=40 \mathrm{nA}$.

The measured SNR shows an optimum SNR of 175 at $U_{\text {acc }}=10 \mathrm{kV}$ for the axial setup and, respectively, 140 at $U_{\text {acc }}=11 \mathrm{kV}$ for the orthogonal setup. This means that the increase in sensitivity is lower than the increase in noise for acceleration voltages above $10 \mathrm{kV}$, which supports the assumption of an increased noise by increased kinetic electron energy. Thus, it is recommended to set the acceleration voltage to its optimum value of $U_{\text {acc }}=10 \mathrm{kV}$ and the emission current to $l_{\text {emis }}=40 \mathrm{nA}$, achieving the maximum SNR of 175 with our current nonradioactive electron capture detector.

Using these optimized electrical parameters, we determined the LoD, based on the measured linear signal increase at low concentrations. The LoD is defined as three times the standard deviation $\sigma$ of the measured noise. For a measuring time of $200 \mathrm{~ms}$, we achieve a LoD of $850 \mathrm{ppt}_{\mathrm{v}}(0.005 \mu \mathrm{g} / \mathrm{l})$ for 1,1,2-trichloroethane.

In addition, we investigated the influence of the carrier gas flow on the LoD. Therefore, the carrier gas flow is increased from $30 \mathrm{mls} / \mathrm{min}$, which is used in the previous described measurements, up to $150 \mathrm{mls} / \mathrm{min}$, while determining the LoD as described before. The resulting LoD are listed in Tab. 1.

Tab. 1: Limit of detection for 1,1,2-trichloroethane for various carrier gas flows. The operating parameters are: $I_{\text {emis }}=40 \mathrm{nA}, U_{a c c}=10 \mathrm{kV}, U_{c o l}=3 \mathrm{~V}$.

\begin{tabular}{|c|c|c|}
\hline \multirow{2}{*}{$\begin{array}{c}\text { Carrier gas } \\
\text { flow } \\
\text { in } \mathrm{mls} / \mathrm{min}\end{array}$} & \multicolumn{2}{|c|}{$\begin{array}{c}\text { Limit of detection } \\
\text { for 1,1,2-trichloroethane }\end{array}$} \\
\cline { 2 - 3 } & in $\mathrm{ppt} \mathrm{v}_{\mathrm{v}}$ & in $\mu \mathrm{g} / \mathrm{l}$ \\
\hline 30 & 850 & 0.005 \\
\hline 60 & 1085 & 0.006 \\
\hline 90 & 1140 & 0.0065 \\
\hline 120 & 1220 & 0.007 \\
\hline 150 & 2930 & 0.016 \\
\hline
\end{tabular}

These results show that the achievable LoD deteriorate up to $2.93 \mathrm{ppb}_{\mathrm{v}}(0.016 \mu \mathrm{g} / \mathrm{l})$ with a carrier gas flow of $150 \mathrm{mls} / \mathrm{min}$. This is due to the fact that a higher flow speed decreases the reaction time between the analyte molecules with the thermalized electrons.

Furthermore, the achievable LoD of our nonradioactive electron source is comparable to the typical limit of detection for 1,1,2-trichloroethane using an ECD with the radioactive counterpart [11]. In addition, typical GC-flows range between $30 \mathrm{mls} / \mathrm{min}$ and $60 \mathrm{mls} / \mathrm{min}[9,12,13]$. Further investigations with an optimized geometry of the ionization chamber are necessary to further improve the achievable LoD, for example at higher carrier gas flows.

\section{Conclusion}

In this work, we present the realization and investigation of an electron capture detector with a non-radioactive electron source. We designed two different ionization chamber configurations with a Faraday plate detector inside and coupled them to our existing non-radioactive electron source. The difference between the two setups is axial gas flow in the first and orthogonal gas flow in the second ionization chamber configuration. The signal to noise ratio (SNR) increases with increasing electron emission current and the highest achievable SNR is reached at the highest possible emission current of $l_{\text {emis }}=40 \mathrm{nA}$ for both setups. This can be explained by the higher number of generated secondary electrons per high kinetic primary electron. However, the SNR of the axial setup is slightly higher than the SNR of the orthogonal setup. Furthermore, the maximum SNR is reached with an acceleration voltage of $U_{\text {acc }}=10 \mathrm{kV}$. Both an increase and a decrease in the acceleration voltage result in a decrease in SNR of both setups. This is due to the fact that 
an increased electron energy leads to an increased noise because of the statistical behavior of the ionization process. Therefore, we found the optimum operating parameters for our non-radioactive electron source, which is the base for a highly sensitive non-radioactive electron capture detector. Furthermore, it is recommended to use the axial gas flow setup, because its SNR is slightly higher than the SNR of the orthogonal gas flow setup, leading to lower limits of detection.

Finally, the limit of detection for 1,1,2trichloroethane was determined to $850 \mathrm{ppt}_{\mathrm{v}}$ $(0.005 \mu \mathrm{g} / \mathrm{l})$ with the axial setup at the previously described optimal values and a constant carrier gas flow of $30 \mathrm{mls} / \mathrm{min}$. This limit of detection is comparable to the typical limit of detection using an ECD with a radioactive ionization source.

Although our described non-radioactive ECD is already comparable to the widely used radioactive ECDs, future investigations and developments can possibly improve the analytical performance significantly. For example, the development of a transimpedance amplifier with an increased measuring range allows a more detailed investigation of the influence of an increased collector voltage on the achievable SNR and the linearity of the detector response. In addition, the implementation of a pulsed mode can also improve the linearity and the dynamic range of the detector response. Finally, the investigation of reduced carrier gas flows are of particular interest, because many modern gas chromatographs work with gas flows around $10 \mathrm{mls} / \mathrm{min}$ and less.

\section{References}

[1] J. E. Lovelock, S. R. Lipsky, J. Am. Chem. Soc. 82, 431-433 (1960); doi: 10.1021/ja01487a045.

[2] R. J. Maggs, P. L. Joynes, A. J. Davies, J. E. Lovelock, Anal. Chem. 43, 1966-1971 (1971); doi: 10.1021/ac60308a014.

[3] W. E. Wentworth, E. D. D'Sa, H. Cai, S. Stearns, J. Chromatogr. Sci. 30, 478-485 (1992); doi: 10.1093/chromsci/30.12.478.

[4] P. Cochems, A. T. Kirk, E. Bunert, M. Runge, P. Goncalves, S. Zimmermann, Rev. Sci. Instrum. 86, 65102 (2015); doi:

10.1063/1.4921707.

[5] E. Bunert, A. Heptner, T. Reinecke, A. T. Kirk, S. Zimmermann, Review of Scientific Instruments 88, 24102 (2017); doi: 10.1063/1.4976021.

[6] H. Cai, W. E. Wentworth, S. D. Stearns, Anal. Chem. 68, 1233-1244 (1996); doi: 10.1021/ac951047j.

[7] P. L. Gobby, E. P. Grimsrud, S. W. Warden, Anal. Chem. 52, 473-482 (1980); doi: 10.1021/ac50053a023.
[8] P. Cochems, A. Kirk, S. Zimmermann, The Review of scientific instruments 85, 124703 (2014); doi: 10.1063/1.4902854.

[9] P. D. Wagner, P. F. Naumann, R. B. Laravuso, Journal of applied physiology 36, 600-605 (1974).

[10] A. Zlatkis, C.F. Poole, Electron capture: Theory and practice in chromatography, Elsevier Scientific Pub. Co, Amsterdam, New York, 1981.

[11] F. Barani, N. Dell'Amico, L. Griffone, M. Santoro, C. Tarabella, Journal of Chromatographic Science 44, 625-630 (2006); doi: $10.1093 / \mathrm{chromsci} / 44.10 .625$.

[12] V. Zitko, O. Hutzinger, S. Safe, Bulletin of environmental contamination and toxicology 6 , 160-163 (1971).

[13] S. Kapila, W. A. Aue, J. Chrom. A 108, 13-21 (1975); doi: 10.1016/S0021-9673(00)97484-3. 